\title{
ARTÍCULOS
}

\section{Wood anatomical traits of the Araucaria Forest, Southern Brazil}

\author{
Anatomía de la madera del bosque de araucaria, sur de Brasil
}

\author{
Patricia Soffiatti a*, Maria Regina Torres Boeger a, Silvana Nisgoski ${ }^{\text {b }}$, Felipe Kauai ${ }^{a}$ \\ *Corresponding Author: ${ }^{a}$ Universidade Federal do Paraná, Departamento de Botânica, CxP 19031, CEP81531-990, \\ Curitiba, Brazil, phone: +55 41 3361-1631, psoffiatti.ufpr@gmail.com \\ ${ }^{\mathrm{b}}$ Universidade Federal do Paraná, Departamento de Engenharia Florestal, Curitiba, Brazil.
}

\begin{abstract}
SUMMARY
The goal of the present study was to find a pattern regarding wood anatomical features for the Araucaria Forest. For that, we studied the wood anatomy of 17 tree species characteristics of this forest formation of Southern Brazil. The species were selected based on the amplified importance value. Wood samples of three individuals per species were collected and prepared according to standard wood anatomical techniques. Most of the species can be grouped according to the presence of the following features: visible growth rings, diffuse porosity, absence of any typical vessel arrangement, simple perforation plate, simple to diminute bordered pits in fibers, little axial parenchyma, heterogeneous rays. The Grouping Analysis of qualitative and quantitative characters groups the species together, but two are distinct from the others: Cinnamodendron dinisii and Roupala montana. Principal Component Analysis explained $69 \%$ of the total variance, influenced by rays height and width, vessel element and fiber length, separating Cinnamodendron dinisii and Roupala montana from the others. Results corroborated ecological wood anatomical patterns observed for other species in other tropical and subtropical vegetation formations occurring in higher altitudes and latitudes, where the species can be characterized by the presence of visible growth rings, predominantly solitary vessels, simple perforation plates and little axial parenchyma.
\end{abstract}

Key words: altitude, diffuse-porous wood, ecological wood anatomy, growth rings.

\section{RESUMEN}

Se presenta el estudio anatómico de la madera de 17 especies de árboles características de una formación de bosque de araucaria, en el sur de Brasil. Se colectaron 17 especies, basado en el valor de importancia ampliado. Muestras de madera de tres individuos por especie fueron recolectadas, y preparadas de acuerdo con técnicas usuales empleadas en anatomía de la madera. La mayoría de las especies se pueden agrupar para la presencia de las siguientes características: anillos crecimiento visibles, porosidad difusa, los vasos no presentaron patrones específicos de agrupamientos, platina de perforación simples, punteaduras diminutas a bordeadas en las fibras, poco parénquima axial, radios heterogéneos. El análisis de agrupamiento de los caracteres cualitativos y cuantitativos de las especies se las agruparon juntas, pero dos son distintos de los demás: Cinnamodendron dinnisii y Roupala montana. El análisis de componentes principales explicó $69 \%$ de la varianza total, influido por la altura y ancho de los radios, la longitud de los elementos de vasos y de las fibras, separando Cinnamodendron dennisii y Roupala montana de los demás. Los resultados corroboraron los patrones anatómicos ecológicos de la madera observados en otras formaciones vegetales tropicales y subtropicales, en mayor altitud y latitud.

Palabras clave: altitud, anatomía ecológica de la madera, anillos de crecimiento, porosidad difusa.

\section{INTRODUCTION}

Several investigations have been conducted in order to correlate anatomical wood traits to environmental characteristics. Many authors demonstrated that several wood features respond to abiotic conditions such as: variations in diameter, length and frequency of vessels (Carlquist 2001, Luchi 2004, Bosio et al. 2010, Melo Júnior et al. 2011); length and width of fiber walls (Luchi 2004); presence of growth rings (Alves and Angyalossy 2000, Luchi 2004, Barros et al. 2006).
The structural diversity found for secondary xylem allows direct correlations between wood anatomy and different environment conditions; some trends were established (Carlquist 2001, Baas and Schweingruber 1987, Wheeler et al. 2007).

For Brazilian flora there are a few studies focusing on ecological wood anatomy. Alves and Angyalossy (2000, 2002) studied wood traits for several Brazilian regions and showed some trends. Barros et al. (2006) observed some features characterizing an area of Atlantic Forest forma- 
tion. Bosio et al. (2010) studied Miconia sellowiana wood occurring in three forest formations of Atlantic forest in Southern Brazil. Sonsin et al. (2012) compared species of Cerrado and Gallery forest.

Although some studies on wood anatomical ecological traits in Brazilian biomes have been carried out, literature is still scarce considering the richness of Brazilian flora. The goal of the present study is to find a pattern regarding wood anatomical features for the Araucaria Forest. For that, we studied the wood anatomy of 17 tree species characteristics of this forest formation of Southern Brazil. The studied group showed in common the presence of growth rings, diffuse porous wood and little axial parenchyma; patterns common to higher altitude and latitude subtropical and tropical forest formations.

\section{METHODS}

Specimens were collected in two fragments of Araucaria Forest in Curitiba, Paraná state, Brazil. The species selection was based on the amplied importance value (AIV) obtained in Rondon Neto et al. (2002) for this formation. One is known as "Bosque da Engenharia Florestal" situated in the campus of Universidade Federal do Paraná, Campus 3 -Jardim Botânico da Universidade Federal do Paraná (2526'50” S, 49 $\left.{ }^{\circ} 14^{\prime} 16^{\prime \prime} \mathrm{W}\right)$, (Rondon Neto et al. 2002). The other fragment is known as "Reserva Mata Viva", located in Centro Politécnico da Universidade Federal do Pa-

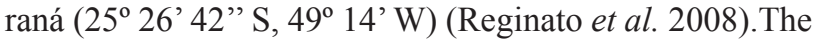
climate of the region is humid subtropical, characterized by hot, usually humid summers; climate classification $\mathrm{Cfb}$ according to Köppen (Maack 2002). Annual mean temperature in hot and cold months is under $22^{\circ} \mathrm{C}$ and $18^{\circ} \mathrm{C}$, respectively. The mean annual temperature oscillates between $15{ }^{\circ} \mathrm{C}$ and $18{ }^{\circ} \mathrm{C}$, with frequent frosts in winter periods. The annual means of relative air humidity and precipitation in this region are $85 \%$ and $1,400-1,600 \mathrm{~mm}$, respectively, with a hydric index varying from 60 up to 100 , with no occurrence of hydric deficit along the year (IAPAR 2000).

Samples from the outer wood of seventeen species (table 1) from mature populations were taken at breast height $(1.30 \mathrm{~m})$. For each species, three individuals were selected, totalizing 51 samples. Samples were sectioned and macerated for light microscopy according to standard techniques in wood anatomy. Terminology for descriptions followed the recommendations of the IAWA List of Microscopic Features for Harwood Identification (IAWA 1989).

For qualitative characteristics, percentages of absolute occurrence of characters were calculated for each species. Measurements were made on the following quantitative characters: vessel diameter, vessel length, fiber length, ray height and width. For each characteristic, 25 measurements per individual were taken. Means and standard deviation are on table 2 .
Table 1. List of studied families and species, respectively. Lista de las familias y de las especies estudiadas, respectivamente.

\begin{tabular}{|c|c|}
\hline Family & Species \\
\hline Anacardiaceae & Schinus terebinthifolia Raddi \\
\hline Asteraceae & $\begin{array}{l}\text { Moquiniastrum polymorphum (Less.) } \\
\text { G. Sancho }\end{array}$ \\
\hline Bignoniaceae & Jacaranda puberula Cham. \\
\hline Canellaceae & Cinnamodendron dinisii Schwacke \\
\hline Euphorbiaceae & Gymnanthes klotzschiana Müll.Arg. \\
\hline Fabaceae & Dalbergia frutescens (Vell.) Britton \\
\hline Lauraceae & Ocotea puberula (Rich.) Nees \\
\hline Malvaceae & Luehea divaricata Mart. et Zucc. \\
\hline \multirow[t]{4}{*}{ Myrtaceae } & Calyptranthes concinna DC. \\
\hline & $\begin{array}{l}\text { Campomanesia xanthocarpa (Mart.) O. } \\
\text { Berg }\end{array}$ \\
\hline & $\begin{array}{l}\text { Myrceugenia miersiana (Gardner) D. } \\
\text { Legrand et Kausel }\end{array}$ \\
\hline & Myrcia hatschbachii D. Legrand \\
\hline Proteaceae & Roupala montana Aubl. \\
\hline Salicaceae & Casearia sylvestris $\mathrm{Sw}$. \\
\hline \multirow[t]{2}{*}{ Sapindaceae } & $\begin{array}{l}\text { Allophylus edulis (A. St.-Hil., A. Juss. et } \\
\text { Cambess.) Hieron. ex Niederl. }\end{array}$ \\
\hline & Cupania vernalis Cambess. \\
\hline Solanaceae & Solanum sanctae-catharinae Dunal \\
\hline
\end{tabular}

Statistical analyses were carried out on the software PAST 2.09 (Hammer et al. 2001). Analyses of grouping for selected qualitative and quantitative data were made, as well as analyses of principal components (PCA) for quantitative data (tables 2, 3). For grouping analyses, Manhattan similarity index was used.

\section{RESULTS}

Wood anatomical descriptions. Distinct growth rings (figure 1A) are present in $88 \%$ of the samples, indistinct growth rings in Schinus terebinthifolia and Roupala montana. Diffuse-porous wood in all samples, without specific arrangement of vessels in $88 \%$ of the samples, except in $R$. montana (tangential vessel orientation) (figure 1B) and Moquiniastrum polymorphum (dendritic arrangement) (figure 1C). Solitary vessels in Campomanesia xanthocar$p a$ and Myrceugenia miersiana (figure 1D); solitary vessels and radial multiples of 2 in Cinnamodendron dinisii; solitary vessels and radial multiples of 2-3 in Dalbergia frutescens, Ocotea puberula, Calyptranthes conccina and Myrcia hatsbachii; solitary vessels and radial multiples of 2-4 in Roupala montana, Casearia sylvestris and Solanum 
Table 2. Mean values, \pm standard deviation and maximum and minimum values (between brackets) of secondary xylem anatomical characteristics of the studied species.

Promedio, \pm desviación estándar y los valores máximo y mínimo (entre paréntesis) de las características anatómicas del xilema secundario de las especies estudiadas.

\begin{tabular}{|c|c|c|c|c|c|}
\hline Species & $\begin{array}{l}\text { Vessel diameter } \\
\qquad(\mu \mathrm{m})\end{array}$ & $\begin{array}{l}\text { Vessel length } \\
\qquad(\mu \mathrm{m})\end{array}$ & $\begin{array}{l}\text { Fiber length } \\
\qquad(\mu \mathrm{m})\end{array}$ & $\begin{array}{l}\text { Ray width } \\
\qquad(\mu \mathrm{m})\end{array}$ & $\begin{array}{l}\text { Ray height } \\
\qquad(\mu \mathrm{m})\end{array}$ \\
\hline Allophylus edulis & $\begin{array}{l}49.09 \pm 10.31 \\
(81.05-32.79)\end{array}$ & $\begin{array}{c}519.46 \pm 86.02 \\
(768.90-358.58)\end{array}$ & $\begin{array}{c}505.62 \pm 277.90 \\
(1100.74-170.77)\end{array}$ & $\begin{array}{c}16.59 \pm 3.28 \\
(26.75-12.35)\end{array}$ & $\begin{array}{c}171.66 \pm 79.24 \\
(407.68-66.46)\end{array}$ \\
\hline $\begin{array}{l}\text { Calyptranthes } \\
\text { conccina }\end{array}$ & $\begin{array}{c}38.82 \pm 8.55 \\
(61.31-30.67)\end{array}$ & $\begin{array}{c}721.89 \pm 130.90 \\
(938.11-376.31)\end{array}$ & $\begin{array}{c}1224.88 \pm 513.49 \\
(3059.52-411.38)\end{array}$ & $\begin{array}{c}18.48 \pm 5.64 \\
(29.70-8.55)\end{array}$ & $\begin{array}{l}253.54 \pm 65.31 \\
(461.3-152.1)\end{array}$ \\
\hline $\begin{array}{l}\text { Campomanesia } \\
\text { xanthocarpa }\end{array}$ & $\begin{array}{c}108.92 \pm 24.15 \\
(139.79-52.48)\end{array}$ & $\begin{array}{c}383.26 \pm 32.15 \\
(423.26-339.92)\end{array}$ & $\begin{array}{c}798.25 \pm 293.73 \\
(1437.68-444.04)\end{array}$ & $\begin{array}{l}26.31 \pm 11.77 \\
(41.82-5.70)\end{array}$ & $\begin{array}{l}246.69 \pm 114.28 \\
(480.86-51.35)\end{array}$ \\
\hline Casearia sylvestris & $\begin{array}{l}52.78 \pm 12.14 \\
(74.22-20.15)\end{array}$ & $\begin{array}{l}501.75 \pm 131.14 \\
(758.0-258.31)\end{array}$ & $\begin{array}{l}859.53 \pm 158.20 \\
(1126.05-529.1)\end{array}$ & $\begin{array}{l}38.65 \pm 13.31 \\
(60.75-11.26)\end{array}$ & $\begin{array}{c}555.84 \pm 200.04 \\
(974.81-175.73)\end{array}$ \\
\hline $\begin{array}{l}\text { Cinnamodendron } \\
\text { dinisii }\end{array}$ & $\begin{array}{l}42.92 \pm 11.65 \\
(60.82-20.04)\end{array}$ & $\begin{array}{c}1463.47 \pm 183.91 \\
(1757.38-1152.55)\end{array}$ & $\begin{array}{c}1286.06 \pm 434.68 \\
(2095.56-538.86)\end{array}$ & $\begin{array}{l}256.56 \pm 4.69 \\
(25.52-10.50)\end{array}$ & $\begin{array}{l}256.56 \pm 161.56 \\
(654.99-65.67)\end{array}$ \\
\hline Cupania vernalis & $\begin{array}{c}89.58 \pm 19.27 \\
(133.47-59.89)\end{array}$ & $\begin{array}{c}470.38 \pm 96.85 \\
(624.84-290.69)\end{array}$ & $\begin{array}{c}798.35 \pm 141.09 \\
(1231.93-590.24)\end{array}$ & $\begin{array}{c}11.17 \pm 3.16 \\
(18.55-7.81)\end{array}$ & $\begin{array}{c}129.61 \pm 61.41 \\
(280.44-57.29)\end{array}$ \\
\hline Dalbergia frutescens & $\begin{array}{c}136.5 \pm 59.54 \\
(250.66-63.46)\end{array}$ & $\begin{array}{c}166.62 \pm 32.64 \\
(222.21-94.52)\end{array}$ & $\begin{array}{c}994.76 \pm 268.24 \\
(1573.69-655.63)\end{array}$ & $\begin{array}{l}23.63 \pm 8.91 \\
(46.52-9.70)\end{array}$ & $\begin{array}{c}117.76 \pm 27.20 \\
(165.37-59.54)\end{array}$ \\
\hline $\begin{array}{l}\text { Gymnanthes } \\
\text { klotzschiana }\end{array}$ & $\begin{array}{l}63.12 \pm 16.37 \\
(83.01-22.58)\end{array}$ & $\begin{array}{c}565.19 \pm 119.08 \\
(818.92-297.76)\end{array}$ & $\begin{array}{l}1380.56 \pm 326.42 \\
(2220.32-801.84)\end{array}$ & $\begin{array}{c}17.35 \pm 4.20 \\
(29.62-11.28)\end{array}$ & $\begin{array}{c}295.21 \pm 129.23 \\
(629.38-114.45)\end{array}$ \\
\hline Jacaranda puberula & $\begin{array}{c}70.37 \pm 21.46 \\
(113.92-26.05)\end{array}$ & $\begin{array}{c}380.91 \pm 75.20 \\
(524.01-220.48)\end{array}$ & $\begin{array}{c}962.51 \pm 147.01 \\
(1362.42-714.08)\end{array}$ & $\begin{array}{c}22.61 \pm 6.94 \\
(40.79-14.75)\end{array}$ & $\begin{array}{c}253.53 \pm 88.84 \\
(437.66-120.66)\end{array}$ \\
\hline Luehea divaricata & $\begin{array}{c}78.83 \pm 15.86 \\
(120.10-53.35)\end{array}$ & $\begin{array}{c}290.32 \pm 38.56 \\
(368.47-208.47)\end{array}$ & $\begin{array}{c}858.14 \pm 175.35 \\
(1178.29-464.96)\end{array}$ & $\begin{array}{c}23.89 \pm 9.24 \\
(51.22-10.45)\end{array}$ & $\begin{array}{c}238.91 \pm 27.73 \\
(283.24-171.88)\end{array}$ \\
\hline $\begin{array}{l}\text { Moquiniastrum } \\
\text { polymorphum }\end{array}$ & $\begin{array}{l}44.64 \pm 10.94 \\
(70.09-26.96)\end{array}$ & $\begin{array}{c}266.67 \pm 36.95 \\
(356.47-188.24)\end{array}$ & $\begin{array}{c}907.66 \pm 153.26 \\
(1195.38-601.18)\end{array}$ & $\begin{array}{l}44.47 \pm 14.36 \\
(74.69-9.58)\end{array}$ & $\begin{array}{c}198.22 \pm 65.57 \\
(327.71-96.04)\end{array}$ \\
\hline $\begin{array}{l}\text { Myrceugenia } \\
\text { mierciana }\end{array}$ & $\begin{array}{c}119.28 \pm 25.09 \\
(157.11-10.45)\end{array}$ & $\begin{array}{c}537.56 \pm 108.81 \\
(786.55-309.15)\end{array}$ & $\begin{array}{c}1170.12 \pm 329.03 \\
(2395.63-746.11)\end{array}$ & $\begin{array}{c}22.87 \pm 8.60 \\
(39.10-10.45)\end{array}$ & $\begin{array}{l}257.84 \pm 102.00 \\
(502.50-98.09)\end{array}$ \\
\hline Myrcia hatschbachii & $\begin{array}{c}40.08 \pm 4.01 \\
(47.0-32.98)\end{array}$ & $\begin{array}{c}374.27 \pm 104.25 \\
(559.69-152.25)\end{array}$ & $\begin{array}{c}1081.52 \pm 211.38 \\
(1600.69-649.97)\end{array}$ & $\begin{array}{l}22.30 \pm 6.45 \\
(31.26-7.86)\end{array}$ & $\begin{array}{c}417.61 \pm 170.21 \\
(898.69-172.36)\end{array}$ \\
\hline Ocotea puberula & $\begin{array}{c}135.94 \pm 23.91 \\
(183.98-75.08)\end{array}$ & $\begin{array}{c}644.46 \pm 92.14 \\
(857.52-504.33)\end{array}$ & $\begin{array}{c}1078.05 \pm 129.86 \\
(1310.62-840.26)\end{array}$ & $\begin{array}{c}40.37 \pm 8.18 \\
(60.78-20.85)\end{array}$ & $\begin{array}{c}449.78 \pm 154.27 \\
(828.05-215.90)\end{array}$ \\
\hline Roupala montana & $\begin{array}{c}82.74 \pm 18.74 \\
(131.53-53.84)\end{array}$ & $\begin{array}{c}437.55 \pm 95.71 \\
(581.95-243.40)\end{array}$ & $\begin{array}{c}1022.42 \pm 256.01 \\
(1551.84-523.37)\end{array}$ & $\begin{array}{c}762.29 \pm 565.30 \\
(2110.54-355.610)\end{array}$ & $\begin{array}{l}2873.48 \pm 956.31 \\
(3989.88-753.38)\end{array}$ \\
\hline $\begin{array}{l}\text { Schinus } \\
\text { terebinthifolia }\end{array}$ & $\begin{array}{l}43.79 \pm 15.21 \\
(71.12-19.09)\end{array}$ & $\begin{array}{c}336.43 \pm 76.79 \\
(514.95-171.88)\end{array}$ & $\begin{array}{c}662.35 \pm 87.76 \\
(844.90-480.50)\end{array}$ & $\begin{array}{c}25.65 \pm 4.27 \\
(35.68-18.31)\end{array}$ & $\begin{array}{c}272.85 \pm 61.61 \\
(379.73-182.93)\end{array}$ \\
\hline $\begin{array}{l}\text { Solanum sanctae- } \\
\text { catharinae }\end{array}$ & $\begin{array}{l}59.65 \pm 18.48 \\
(93.78-27.89)\end{array}$ & $\begin{array}{c}316.57 \pm 69.85 \\
(502.96-222.47)\end{array}$ & $\begin{array}{c}778.54 \pm 191.53 \\
(1184.83-452.55)\end{array}$ & $\begin{array}{l}29.88 \pm 12.11 \\
(47.81-10.73)\end{array}$ & $\begin{array}{c}296.10 \pm 96.71 \\
(489.58-146.04)\end{array}$ \\
\hline
\end{tabular}


Table 3. PCA - Principal components (PC) and variance percentage of each component.

PCA - Componentes principales y porcentaje de varianza de cada componente.

\begin{tabular}{clc}
\hline PC & Eigenvalue & \% Variance \\
\hline 1 & 2.0415 & 40.82 \\
2 & 1.45199 & 29.04 \\
3 & 1.04753 & 20.95 \\
4 & 0.429916 & 8.59 \\
5 & 0.0295976 & 0.59 \\
\hline
\end{tabular}

sanctae-catharinae; solitary vessels and radial multiples of 2-6 in Jacaranda puberula and Allophylus edulis; solitary vessels and radial multiples of 2-9 in Schinus terebinthifolia, Gymnanthes klotzschiana, Luehea divaricata and Cupania vernalis. Simple perforation plates (figure $1 \mathrm{E})$ in $94 \%$ of the samples, except in Cinnamodendron dinisii (scalariform perforation plate) (figure 2A). Reticulate perforation plates in Calyptranthes conccina and Myrcia hatschbachii (figure 1F). Intervessel pits alternate and bordered (figure 2B) in $88 \%$ of the individuals, except in Cinnamodendron dinisii and Calyptranthes conccina (opposite and bordered) (figure 2A). Vestured pits (figure 2A) in Cinnamodendron dinisii. Septate fibers (figure 2C) in $47 \%$ of the individuals: Ocotea puberula, Calyptranthes conccina, Myrceugenia miersiana, Casearia sylvestris, Allophylus edulis, Cupania vernalis and Solanum sanctaecatharinae. Axial parenchyma in $66 \%$ of the samples, except in Moquiniastrum polymorphum, Jacaranda puberula, Dalbergia frutescens, Ocotea puberula, Roupala montana and Casearia sylvestris, in which vasicentric axial parenchyma is predominant (figure 2F) or confluent (figure 2D). Rays predominantly uniseriate, with some rare biseriate (figure 2F) in Allophylus edulis, from two to five cells of width among uniseriate rays in $88 \%$ of the samples and from one to 38 cells of width in Roupala montana (figure 3A). Aggregate rays in Luehea divaricate (figure $3 \mathrm{~B}$ ); heterogeneous rays (procumbent cells with borders composed of squared to erect cells) (figure 3D) in $88 \%$ of the individuals, except in Cinnamodendron dinisii and $\mathrm{Cu}$ pania vernalis, which have homogeneous rays (procumbent cells only) (figure 3C). Disjunctive parenchyma ray cells (figure 3E) in Calyptranthes conccina, Myrcia hatsbachii and Solanum sanctae-catharinae $65 \%$ of the samples presented cell contents; crystals were present in $47 \%$ of the samples and radial channels were found in Schinus terebinthifolia (figure 3F). Bordered ray-vessel pits in all samples. Diffuse apotracheal parenchyma present in $47 \%$ and apotracheal parenchyma diffuse to aggregate in $53 \%$ of the samples; confluent paratracheal parenchyma (figure
2D) in $12 \%$; scanty (according to the IAWA list, IAWA 1989) paratracheal parenchyma in $65 \%$ and vasicentric paratracheal parenchyma (figure $2 \mathrm{E}$ ) in $12 \%$; vasicentric and confluent paratracheal parenchyma represented in $12 \%$ of the samples.

The selected qualitative data for grouping analyses were: distinct growth ring; diffuse-porous wood; no specific arrangement of vessels; solitary or solitary and multiple vessels; simples perforation plate; reticulate perforation plate; intervessel pits alternate and bordered; bordered ray-vessel pits; bordered to simple fiber pits; septate fibers; diffuse or diffuse to aggregate apotracheal parenchyma; confluent paratracheal parenchyma and vasicentric parenchyma; rays predominantly unisseriate, rare bisseriate; rays with one to five cells of width; rays with one to 38 cells of width; homogeneous rays; disjunctive ray cells; rays cells with content; ray cells with crystals; radial channels.

The grouping analyses for qualitative data (figure 4) did not group the studied species. The grouping analyses for quantitative data (figure 5) separated only Cinnamodendron dinisii and Roupala montana from the rest.

Within PCA analyses, it was observed that anatomical characteristics varied among factors that explained $69 \%$ of the total variance (table 3 ). The axis of component 1 responds for $40 \%$ of the total variance and it is influenced by the height and width of rays. The axis of component 2 responds for $29 \%$ of the total variance and it is influenced by vessel length and fiber length (figure 6).

\section{DISCUSSION}

The studied species have various common characteristics, such as distinct growth ring, diffuse-porous, simple perforation plate, scanty axial parenchyma and heterogeneous rays.

Growth rings were found in $88 \%$ of the individuals, corroborating with Alves and Angyalossy (2000) whose study on ecological trends for wood of various Brazilian species have shown a correlation between growth rings in regions with climatic seasonality or altitude. Barros et al. (2006) observed a high incidence of growth rings studying species of an Atlantic Forest formation of Southeastern Brazil.

In tropical regions, fluctuations in hydric availability might affect cambial activity, thus generating the formation of growth rings (Worbes 1989, Callado et al.2001). Several researches have demonstrated a strong correlation between hydric stress, phenology and cambial activity (Botosso and Tomazello Filho 2001, Callado et al. 2001, Lisi et al. 2008). Although Araucaria Forest does not have a well-demarcated dry season, it shows intervals in which there is a reduction in pluviosity (Bosio et al. 2010), besides a well demarcated cold season with frequent frosts, which may explain the presence of growth rings in the majority of the studied species. 


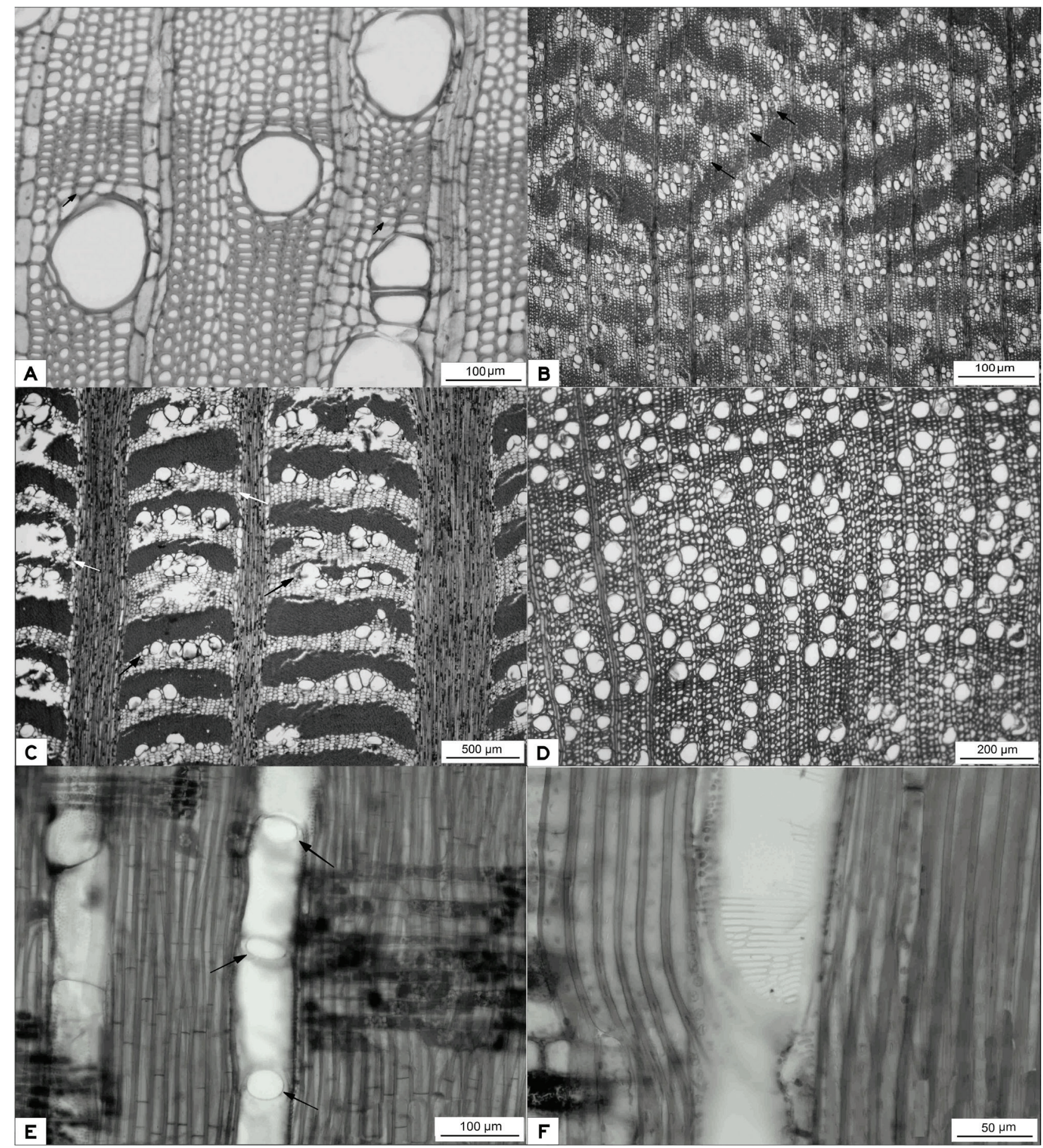

Figure 1. A - Ocotea puberula, cross section (CS). Distinct growth ring demarcated by fibers with thick and tangentially flattened walls. B - Moquiniastrum polymorphum (CS). Arrangement of vessels in diagonal rows (black arrows). C - Roupala montana (CS). Arrangement of vessels in tangential bands (white arrows); confluent parenchyma (black arrows). D - Myrcia haschbachii (CS). Solitary vessels. E - Allophylus edulis, radial longitudinal section (RLS). Simple perforation plate (black arrows). F - Calyptranthes conccina, tangential longitudinal section (TLS). Reticulate perforation plate.

A - Ocotea puberula, sección transversal (ST), anillos de crecimiento distintos demarcados por fibras de paredes gruesas y tangencialmente aplanadas. B - Moquiniastrum polymorphum (ST), disposición de los vasos en filas diagonales (flechas negras). C - Roupala montana (ST), disposición de los vasos en filas tangenciales (flechas blancas); parénquima confluente (flechas blancas). D - Myrcia haschbachii (ST), solitary vessels. E Allophylus edulis, sección longitudinal radial (SLR), platina de perforación simples (flechas negras). F - Calyptranthes conccina, sección longitudinal tangencial (SLT), platina de perforación reticulada. 


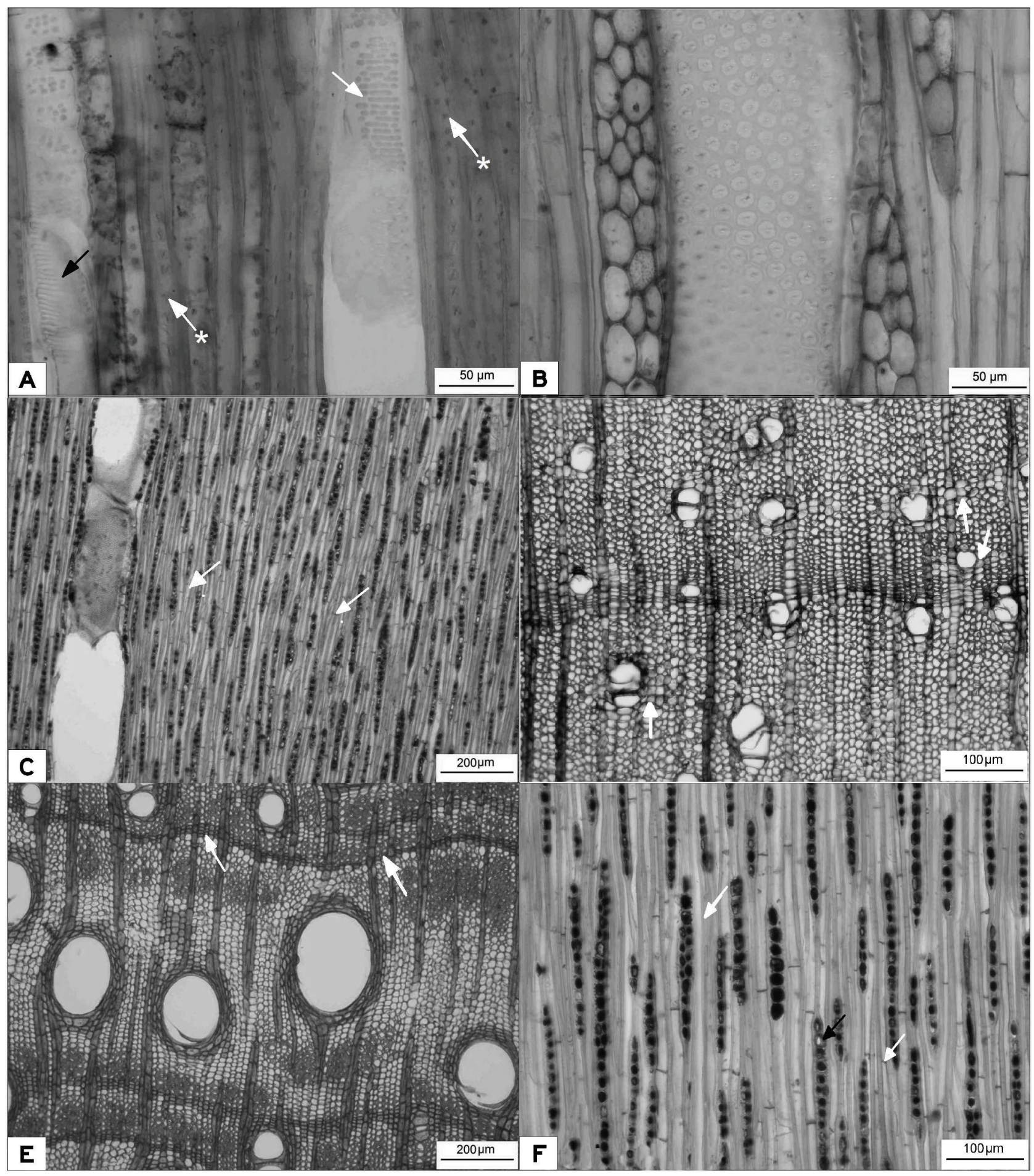

Figure 2. A - Cinnamodendron dinisii, tangential longitudinal section (TLS). Scalariform perforation plate (black arrows); bordered intervessel pits opposite (white arrows); fiber tracheids (white arrow with *). B - Ocotea puberula (TLS). Alternate bordered pits. C - Cupania vernalis (TLS). Septate fibers (white arrows). D - Jacaranda puberula, cross section (CS). Aliform to confluent axial parenchyma (white arrows). E - Dalbergia frutescens (CS). Axial parenchyma vasicentric and in terminal bands (white arrows). F Allophylus edulis (TLS). Unisseriate rays, rare bisseriate; septate fibers (white arrows) and prismatic crystals (black arrows).

A - Cinnamodendron dinisii (SLT), placa de perforación escalariforme (flechas negras); punteaduras intervasculares bordeadas, opuestas (flechas blancas); fibrotraqueidas $(*)$. B - Ocotea puberula (SLT), punteaduras intervasculares bordeadas, alternas. C - Cupania vernalis (SLT), fibras septadas (flechas blancas). D - Jacaranda puberula (ST), parénquima axial aliforme a confluente (flechas blancas). E - Dalbergia frutescens (ST), parénquima axial vasicéntrico y en bandas terminales (flechas blancas). F - Allophylus edulis (SLT), radios uniseriados, raros biseriados; fibras septadas (flechas blancas) y cristales prismáticos (flechas negras). 


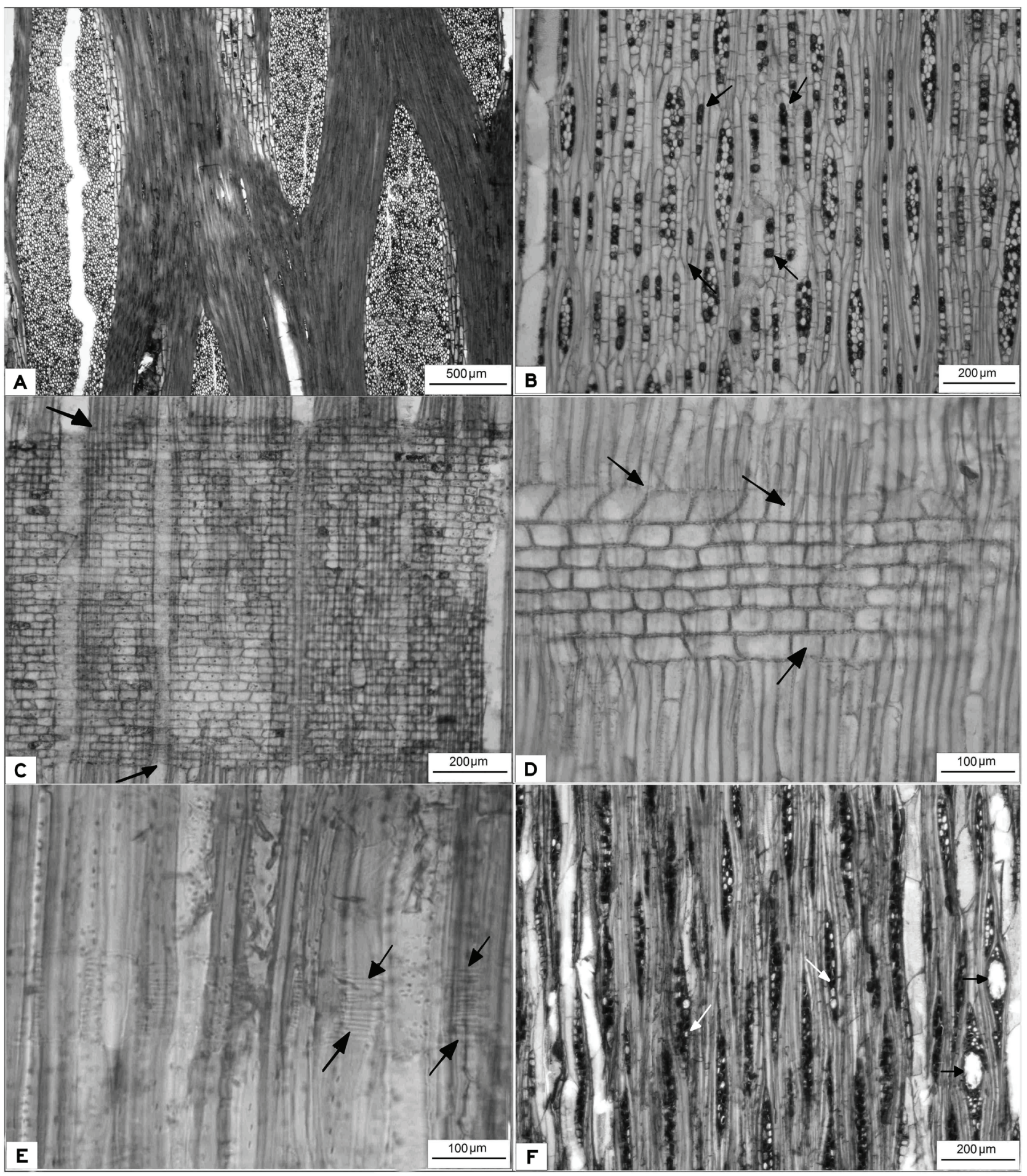

Figure 3. A - Roupala Montana, tangential longitudinal section (TLS). Rays with up to 38 cells of width. B - Luehea divaricate (TLS). Stratified rays. C - Cinnamodendron dinisii, radial longitudinal section (RLS). Homogeneous rays, with procumbent cells only (black arrows). D - Gymnanthes klotzschiana (TLS). Heterogeneous rays with body composed of procumbent cells and margins of squared and erected (black arrows). E - Calyptranthes conccina (TLS). Ray cells with disjunctive cell walls (black arrows) F - Schinus terebinthifolia (TLS). Content inside ray cells (white arrows) and radial channels (black arrows).

A - Roupala montana (SLT), radios multiseriados con 38 células de ancho. B - Luehea divaricate (SLT), radios estratificados. C - Cinnamodendron dinisii (SLR), radios homocelulares, compuestos por células procumbentes (flechas negras). D - Gymnanthes klotzschiana (SLT), radios heterocelulares con un cuerpo de células procumbentes y extremos con células cuadradas o erectas (flechas negras). E - Calyptranthes conccina (SLT), radios con paredes disyuntas (flechas negras). F - Schinus terebinthifolia (SLT), radios con contenidos (flechas blancas) y canales radiales (flechas negras). 


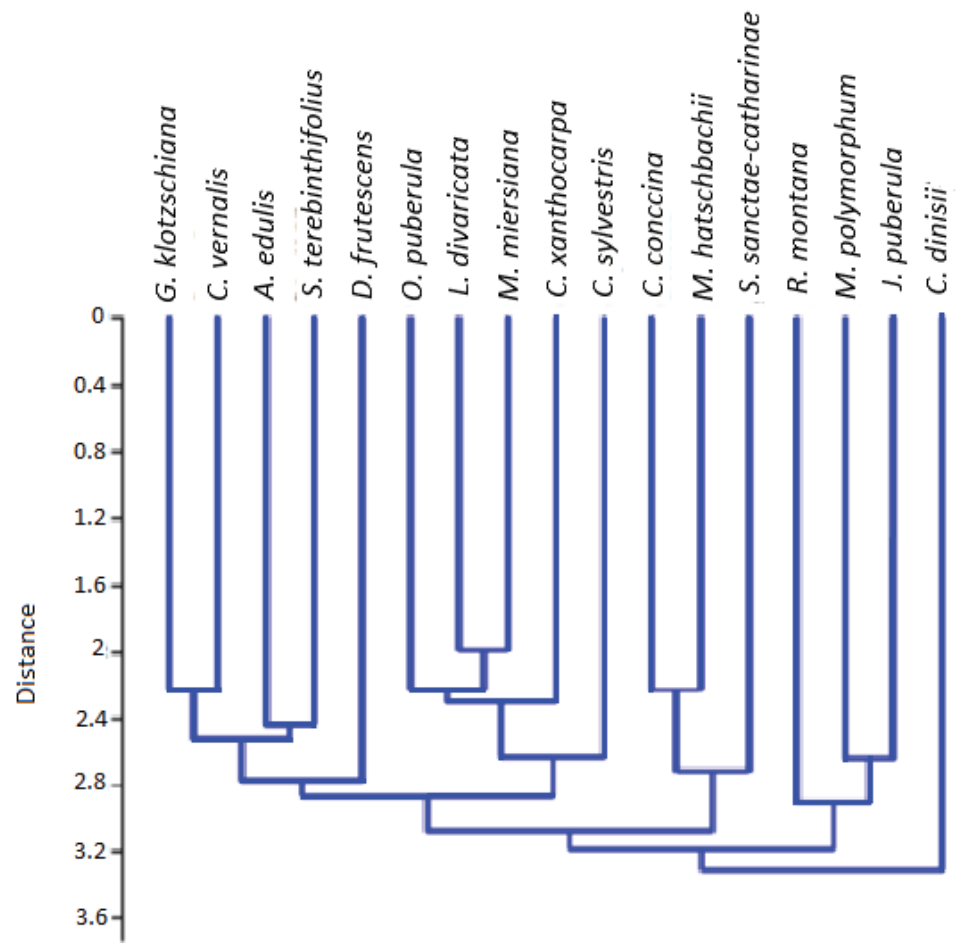

Figure 4. Grouping analysis dendrogram based on Manhattan similarity index of qualitative wood traits.

Dendrograma del análisis de agrupamiento baseado en el índice de similitud de Manhattan de los caracteres cualitativos de la madera.

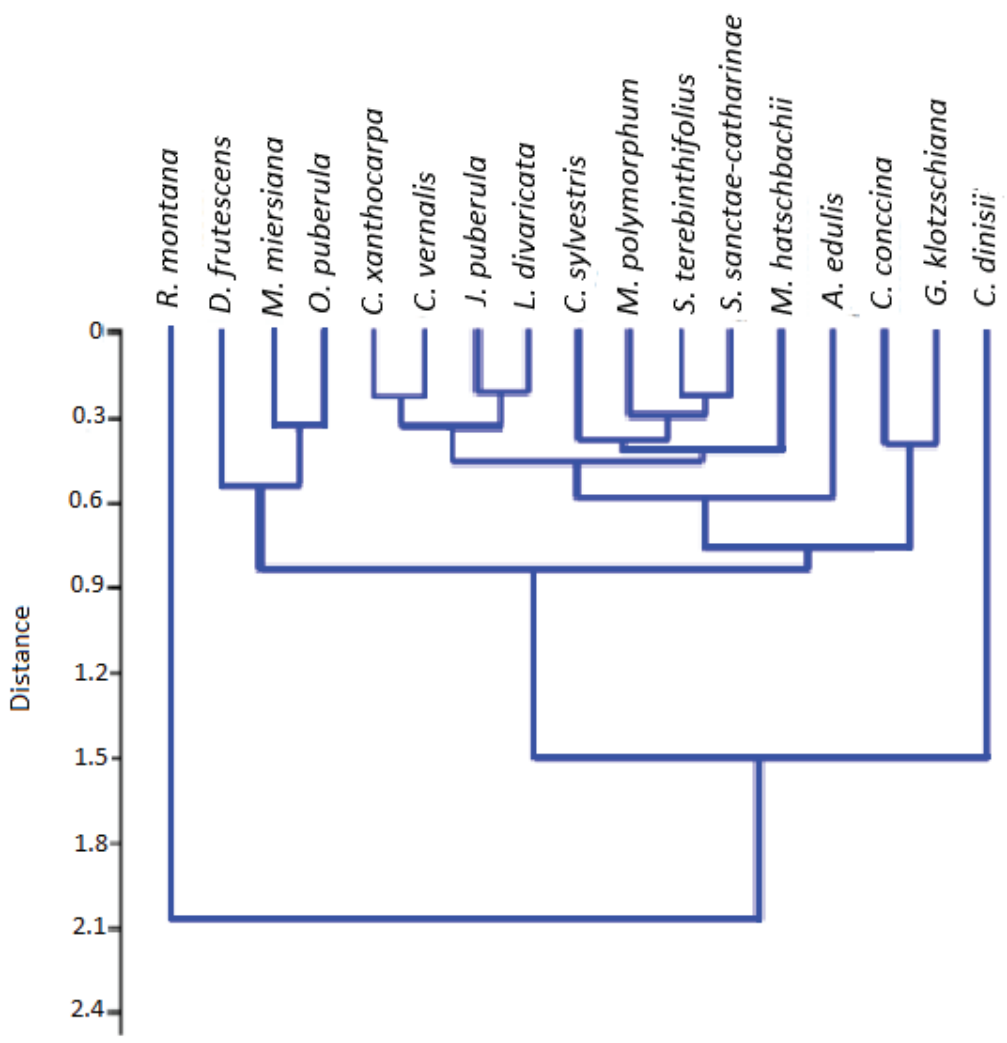

Figure 5. Grouping analyses dendrogram based on Manhattan similarity index of quantitative wood traits (table 2). (cuadro 2).

Dendrograma del análisis de agrupamiento baseado en el índice de similitud de Manhattan de los caracteres cuantitativos de la madera 


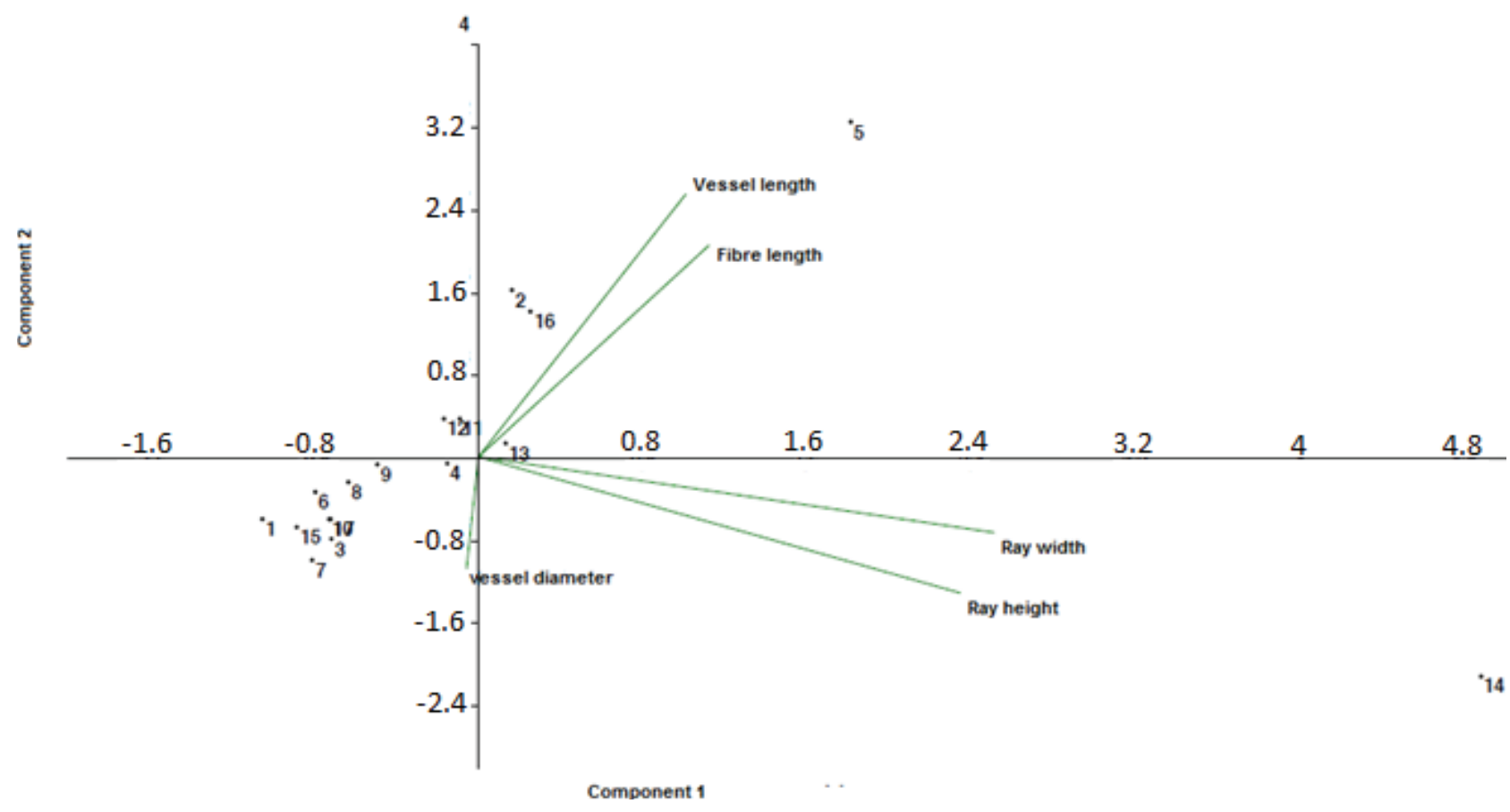

Figure 6. Principal components analyses (PCA) of quantitative wood traits for the studied species: 1. Allophylus edulis, 2. Calyptranthes concinna, 3. Campomanesia xanthocarpa, 4. Casearia sylvestris, 5. Cinnamodendron dinisii, 6. Cupania vernalis, 7. Dalbergia frutescens, 8. Moquiniastrum polymorphum, 9. Jacaranda puberula, 10. Luehea divaricata, 11. Myrceugenia miersiana, 12. Myrcia hatschbachii, 13. Ocotea puberula, 14. Roupala montana, 15. Schinus terebinthifolia, 16. Gymnanthes klotzschiana, 17. Solanum sanctae-catharinae.

Análisis de componentes principales (ACP) de los caracteres cuantitativos de la madera para las especies estudiadas: 1. Allophylus edulis, 2. Calyptranthes concinna, 3. Campomanesia xanthocarpa, 4. Casearia sylvestris, 5. Cinnamodendron dinisii, 6. Cupania vernalis, 7. Dalbergia frutescens, 8. Moquiniastrum polymorphum, 9. Jacaranda puberula, 10. Luehea divaricata, 11. Myrceugenia miersiana, 12. Myrcia hatschbachii, 13. Ocotea puberula, 14. Roupala montana, 15. Schinus terebinthifolia, 16. Gymnanthes klotzschiana, 17. Solanum sanctae-catharinae.

Another common characteristic in all samples is diffuseporous wood, considered a frequent characteristic in most Eudycotiledons (Alves and Angyalossy-Alfonso 2000).

In this study, the mean diameter found is lower than what was found by Barros et al. (2006) for a tropical forest of Brazilian low lands. This can be understood by the fact that although a tropical formation, characterized by a super-humid climate (Bosio et al. 2010), the Araucaria Forest is subject to low temperatures and frequent frosts, which can explain the smaller mean diameter found in vessels. Due to the frost, the presence of narrower vessel elements favors more safety in water conduction in the secondary xylem (Sperry et al. 2008).

Most of the samples did not present any particular vessel arrangement, except for Moquiniastrum polymorphum (Asteraceae) and Roupala montana (Proteaceae). The former presents a diagonal arrangement (dendritic), which in general is not common, restricted to a few genera in a few families that are not phylogenetically close (Carlquist 2001). Roupala montana (Proteaceae) presents vessel arrangement in bands, which according to Carlquist (2001) might be a variation from the diagonal arrangement.
Simple perforation plates were found in $94 \%$ of the studied samples, except in $C$. dinisii, which presents the scalariform type. Cinnamodendron dinisii belongs to $\mathrm{Ca}-$ nellaceae, a basal group within Angiosperms. The presence of scalariform perforation plates is a character considered plesiomorphic considering the evolutionary trends for secondary xylem, occurring in some basal lineages (Carlquist 2001). Barros et al. (2006) observed the presence of simple perforation plates in $96 \%$ of the studied species in another Atlantic formation, corroborating with the high index found in this study. Simple perforation plates are regarded as a common feature for most flowering plants (Wheeler et al. 2007). On the other hand, in Myrtaceae, Calyptranthes conccina and Myrcia hatschbachii, besides the simple perforation plates it was also found the reticulate type. For Myrtaceae it was already noticed the presence of both features (Schmid and Baas 1984).

Axial parenchyma predominant type in this study is the diffuse, diffuse in aggregates and scanty. According to literature, there is a trend to species that occur in colder regions to have little axial parenchyma, whereas in warmer regions it is more abundant (Alvesand Angyalossy- 
Alfonso 2002), corroborated by the present results. The Araucaria Forest occurs in higher latitudes with frequent cold in winter. Besides, almost $70 \%$ of the studied species that have little axial parenchyma also have septate fibers. These are living cells assuming a similar function to the axial parenchyma in storage, representing an alternative strategy (Carlquist 2001).

Only one species presented exclusively unisseriate rays, Allophylus edulis (Sapindaceae), which is a characteristic of the family (Carlquist 2001). The other species presented a superior variation of ray width. The species with wider rays is Roupala montana, a Proteaceae, where very wide rays is a typical characteristic (IAWA 1989).

Heterogeneous rays are predominant in all individuals, as observed by Alves and Angyalossy-Alfonso (2002) and Barros et al. (2006) who found the majority of heterogeneous rays predominant in species from Southern Brazil. However, the present data does not corroborate with other studies. Fahn et al. (1986) found homogeneous rays more common in Israel flora, correlating its presence to both dry and wet environments. Accordingly, this parameter is controversial and it cannot be related to any particular ecological trend. Wheeler et al. (2007) concluded that, apparently, there are no ecological trends that may be associated to ray features.

Disjunctive ray cells were observed in Calyptranthes conccina, Myrcia hatschbachii and Solanum sanctaecatharinae. Disjunctive cells of axial or radial parenchyma are not a well-studied characteristic (Kitin et al. 2009), but it was already related to some families, as Buxaceae, Cornaceae, Ericaceae and Rosaceae (Richter and Dallwitz 2000), Myrtaceae (Carlquist 2001) and Santalaceae (Kitin et al. 2009), considered more frequent in species from tropical regions (Richter and Dallwitz 2000). Its origin might be related to mechanical forces generated by the rearrangement of cells during differentiation and development of secondary xylem, however little is known about its function (Kitin et al. 2009).

The grouping analyses using qualitative data (figure 4) showed that the species do group together. However, the grouping analyses using quantitative data (figure 5) demonstrated that Cinnamodendron dinisii and Roupala montana are very distinct from the other species. Cinnamodendron dinisii was separated because of its larger vessels and fibers length while $R$. montana for its far largest rays height and width.

PCA analyses demonstrated that anatomical characteristics varied among factors that explained $69 \%$ of the total variance. The component of axis 1 responded for $40 \%$ of the total variance, influenced by rays height and width. The component of axis 2 responded for $29 \%$ of the total variance, influenced by vessel and fiber length. The component 1 separated Roupala montana by its very wide and tall rays, that are far larger when compared to the other species. It also corroborates the result of the grouping analyses based on quantitative traits. It also separa- ted Cinnamodendron dinisii that appears isolated from the other species, due to its very wide rays, also agreeing with grouping analysis results. The component 2 separated $C$. dinisii, Calyptranthes conccinaand Gymnanthes klotzschiana based on the longer vessels and fibers. C. dinisii stands out for the highest values for vessel length when compared to C. conccina and G. klotzschiana. The other species remained grouped, demonstrating that quantitative characteristics are common to the majority of them.

\section{CONCLUSIONS}

The qualitative characters found in this study corroborate with the patterns observed in some ecological studies regarding secondary xylem anatomy, concerning tropical and subtropical vegetation formations located in regions with higher altitude and latitude, such as the presence of distinct growth rings, diffuse-porous vessels and little axial parenchyma. Results showed that only a few species could be separated based on features that are more related to their historical relationships, such as Cinnamodendron dinisii and Roupala montana, due to a strong phylogenetic signal represented by typical wood traits correlated to the taxonomical groups which they belong to. Most of the species could be grouped by several of the quantitative studied traits, indicating the presence of a strong environmental filter. Exceptions are Calyptranthes conccina and Gymnanthes klotzschiana, which stand out from the others. Further studies are welcome in order to broaden the sampling and achieving a more accurate picture of wood trends in this Atlantic Forest formation.

\section{REFERENCES}

Alves ES, V Angyalossy-Alfonso. 2000. Ecological trends in the wood anatomy of some Brazilian species. 1. Growth rings and vessels. IAWA Journal 20(1):3-30.

Alves ES, V Angyalossy-Alfonso. 2002. Ecological trends in the wood anatomy of some Brazilian species. 2. Axial parenchyma, rays and fibres. IAWA Journal 23(4):391-418.

Baas P, FH Schweingruber. 1987. Ecological trends in the wood anatomy of trees, schrubs and climbers from Europe. IAWA Bulletin 8:245- 274.

Barros CF, ML Marcon-Ferreira, CH Callado, HRP Lima,M Cunha,O Marquete, CG Costa. 2006. Tendências ecológicas na anatomia da madeira de espécies da comunidade arbórea da reserva biológica de poço das antas, Rio de Janeiro, Brasil. Rodriguésia 57(3):443-460.

Botosso PC, M Tomazello-Filho. 2001. Aplicação de faixas dendrométricas na dendrocronologia: avaliação da taxa e do ritmo de crescimento do tronco de árvores tropicais e subtropicais. In Maia NB, HL Martos, W Barrela eds. Indicadores ambientais: Conceitos e aplicações.São Paulo, Brazil. EDUC. p. 145-171.

Bosio F, P Soffiatti, MRT Boeger. 2010. Ecological wood anatomy of Miconia sellowiana (Melastomataceae) in three vegetation types of Paraná state, Brazil. IAWA Jounal 31(2):179-190. 
Callado CH, SJ Silva Neto, FR Scarano, CF Barros, CG Costa. 2001. Anatomical features of growth rings in flood-prone trees of the Atlantic Rain Forest in Rio de Janeiro, Brazil. IAWA Journal 22(1):29-42.

Carlquist S. 2001. Comparative wood anatomy. Systematic, ecological and evolutionary aspects of dicotyledon wood. Berlin, Germany. Springer-Verlag. 448 p.

Fahn A, E Werker, P Baas. 1986. Wood anatomy and identification of trees and shrubs from Israel and Adjacent Regions. Jerusalem, Israel. The Israel Academy of Sciences and Humanities. 221 p.

Hammer Ø, DAT Harper, PD Ryan. 2001. PAST: Paleontological statistics software package for education and data analysis. Palaeontologia Electronica 4(1): 9 p. http://palaeo-electronica.org/2001_1/past/issue1_01.htm

IAPAR (Instituto Agronômico do Paraná, BR). 2000. Cartas climáticas básicas do Estado do Paraná. Londrina. IAPAR. Available in http://www.iapar.br/modules/conteudo/conteudo.php? conteudo $=677$

IAWA (International Association of Wood Anatomists, NL). 1989. IAWA list of microscopic features for hardwood identification. IAWA Bulletin 10:219-332.

Kitin P, H Beeckman, T Fuji, R Funada, S Noshiro, H Abe. 2009. What is disjunctive xylem parenchyma? A case study of the african tropical hardwood Okoubaka aubrevillei (Santalaceae). American Journal of Botany 96(8):1399-1408.

Lisi CS, M Tomazello-Filho, PC Botosso, FA Roig, VRB Maria, L Ferreira-Fedele, ARA Voigt. 2008. Tree-ring formation, radial increment periodicity, and phenology of tree species from a seasonal semi-deciduous forest in southeast Brazil. IAWA Journal 29(2):189-207.

Luchi A.E. 2004. Anatomia do lenho de Croton urucurana Baill. (Euphorbiaceae) de solos com diferentes níveis de umidade. Revista Brasileira de Botânica 27(2):271-280.
Maack R. 2012. Geografia física do Estado do Paraná. Curitiba, Brazil. Imprensa Oficial. 526 p.

Melo Júnior JCF, G Ceccantini,C Bona. 2011. Anatomia ecológica do lenho de Copaifera langsdorffii Desf. (Leguminosae) distribuída em diferentes condições edáficas do cerrado sul-brasileiro. Iheringia 66(2):189-200.

Richter HG,MJ Dallwitz. 2000. Commercial timber: description, illustrations, identification, and information retrieval. Available in http://delta-intkey.com/wood/pt/www/chars.htm.

Reginato M, FB Matos, GS Lindoso, CMF Souza, JA Prevedello, JW Morais, PHL Evangelista. 2008. A vegetação na Reserva Mata Viva, Curitiba, Paraná, Brasil. Acta Biologica Paranaense 37(3, 4):229-252.

Rondon Neto RM, C Kozera, RR Andrade, AT Cecy, AP Hummes, E Fritzons, MVW Caldeira, MNM Maciel, MKF Souza. 2002. Caracterização florística e estrutural de um fragmento de Floresta Ombrófila Mista, em Curitiba, PR Brasil. Floresta 32(1):3-16.

Schmid R, P Baas. 1984. The occurrence of scalariform perforation plates and helical vessel wall thickenings in wood of Myrtaceae. IAWA Bulletin 5(3):197-215.

Sonsin JO, P Gasson, CF Barros, CR Marcati. 2012. A comparison of the wood anatomy of 11 species from two cerrado habitats (cerrado s.s. and adjacent gallery forest). Botanical Journal of the Linnean Society 170:257-276.

Sperry JS, FC Meinzer, KA McCulloh. 2008. Safety and efficiency conflicts in hydraulic architecture: scaling from tissues to trees. Plant, Cell \& Environment 31(5):632-645.

Wheeler EA, P Baas, S Rodgers. 2007. Variations in dicot wood anatomy: a global analysis based on the insidewood database. IAWA Journal 28(3):229-258.

Worbes M. 1989.Growth rings, increment and age of trees in inundation forests, savannas and a mountain forest in the Neotropics. IAWA Bulletin 10(2):109-122. 
